\title{
Oclusión temporal de arterias ilíacas internas en cesárea-histerectomía por placenta acreta: enfoque multidisciplinario
}

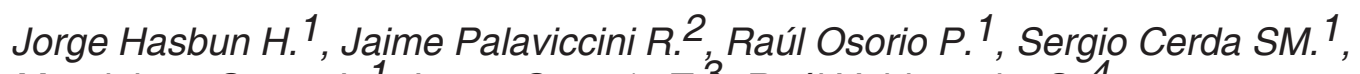 \\ Magdalena Castro L. ${ }^{1}$, Laura Carreño T. 3 , Raúl Valdevenito S. 4 \\ 1 Departamento de Obstetricia y Ginecología, ${ }^{2}$ Departamento de Radiología, ${ }^{3}$ Departamento de Anatomía Patológica, \\ 4 Departamento de Urología. Hospital Clínico Universidad de Chile.
}

\section{RESUMEN}

Objetivo: Evaluar la eficacia y resultados del tratamiento de la placenta acreta previa central en un caso clínico. Durante el embarazo los métodos diagnósticos por imágenes (ultrasonido y resonancia nuclear magnética) precisaron la localización y penetración de la placenta en la pared uterina. En el período inmediato antes del parto, para disminuir la hemorragia, evitar la hipotensión materna y facilitar el procedimiento quirúrgico, se colocaron balones intraarteriales en ambas ilíacas internas por radiólogo intervencionista, catéteres para monitoreo hemodinámico invasivo por anestesiólogo y catéteres ureterales por urólogo. La resolución del parto fue por cesárea-histerectomía sin remoción de la placenta. El caso correspondió a una paciente de 32 años con cesárea en dos partos anteriores, metrorragia episódica y que fue interrumpida en la semana 36. Durante toda la intervención permaneció hemodinámicamente estable requiriendo 1 unidad de glóbulos rojos, 4 litros entre coloides y cristaloides y el posoperatorio fue sin incidentes. El examen histopatológico demostró percretismo de la pared uterina sin invasión vesical, concordante con las imágenes de lagunas vasculares y la citoscopia. Conclusión: El caso demuestra el beneficio del tratamiento especializado multidisciplinario de embarazos con complicaciones graves como la placenta previa con diferentes grados de acretismo.

\section{PALABRAS CLAVE: Placenta, acretismo, incretismo, percretismo}

\section{SUMMARY}

Objective: To report the treatment of a clinical case with central placenta previa accreta. During pregnancy image diagnosis (ultrasound and magnetic resonance imaging) revealed localization and penetration of the placenta into the uterine wall. Just before delivery to reduce the bloss loss and avoid maternal hypotension and facilitate surgery, intra arterial balloons were placed on both hypogastric arteries by the radiologist; intravascular monitoring was installed by anesthesist and urologist put in ureteral catheters. The delivery was by cesarean section and followed by histerectomy leaving the placenta in situ. The case correspond to a woman of 32 years old that had 2 previous cesarean section. During this pregnancy bled a few times and was delivered at weeks 36th. During surgery she was stable, requiring one unit of red blood cells and four liters of coloids and crystaloids. Post-operative course was with no problems. The pathologist report a placenta percreta with no bladder involvement, agreeing with the vascular lakes image and the cystoscopy. 
Conclusion: This case reveals the benefits of multidisciplinary approach to manage severe complications of pregnancy such as placenta previa and the degree of accretism.

\section{KEY WORDS: Placenta, accretism, incretism, percretism}

\section{INTRODUCCIÓN}

El parto quirúrgico en la placenta acreta tiene riesgos de hemorragia, shock hipovolémico, coagulopatía, daño urológico e intestinal, infecciones, complicaciones postoperatorias, morbilidad grave y muerte materna $(1,2)$.

La prevención de estas complicaciones es un objetivo prioritario por el aumento del acretismo placentario en las últimas décadas (3). El manejo actual tiene tres avances importantes: 1 . Un mejor diagnóstico antenatal por imágenes con detección oportuna e interrupción programada, que tiene una incidencia de hemorragia significativamente menor que el parto de urgencia $(4,5)$. 2. Un enfoque multidisciplinario con otros especialistas (radiólogo intervencionista, urólogo, anestesiólogo) que aportan técnicas específicas que permiten disminuir el sangrado, las transfusiones sanguíneas y la incidencia de complicaciones (6). 3. Variaciones de la técnica quirúrgica: tipo de laparotomía e histerotomía, asistencia del alumbramiento, ligaduras vasculares selectivas, oclusión temporal de aorta y cambios anestesiológicos (7-11).

Un procedimiento en evaluación es la oclusión de las arterias ilíacas con balones inflados postparto, apenas ligado el cordón umbilical, suprimiendo el flujo arterial uterino durante la cesárea-histerectomía, minimizando el sangrado intraoperatorio (12) y la eventual embolización si la hemorragia persiste (13).

El objetivo de esta comunicación es describir el caso de una paciente con placenta previa central acreta que con manejo multidisciplinario se adoptó la técnica de oclusión arterial temporal con balón previo a la cesárea-histerectomía.

\section{Caso clínico}

Paciente de 33 años, con antecedentes de dos cesáreas anteriores, con diagnóstico de placenta previa central desde la semana 22 , sin migración posterior, metrorragia episódica y signos ultrasónicos de acretismo (lagunas placentarias grado 2, hipervascularización subplacentaria y del tabique vesicouterino y resonancia nuclear magnética con- firmatoria (Figuras 1,2,3), motivo por lo que se planificó la interrupción de la gestación en la semana 36.

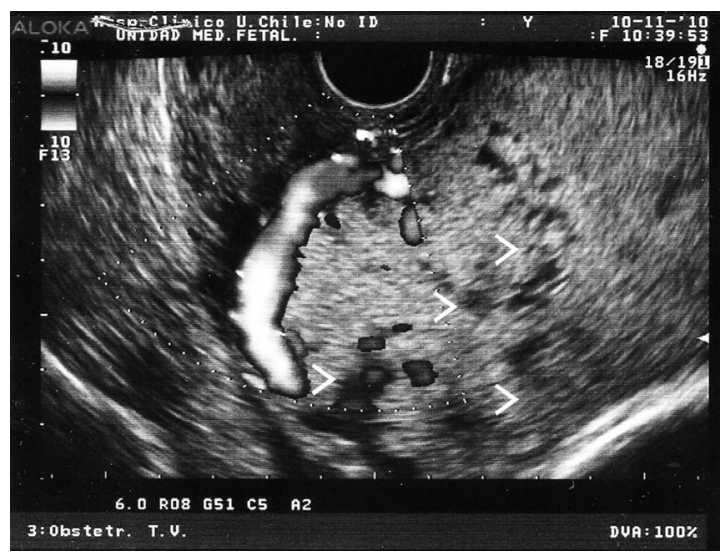

Figura 1. Ultrasonido en placenta acreta con lagunas vasculares numerosas e irregulares, tipo 2 (puntas de flecha) e hipervascularización subplacentaria sobre el cuello.

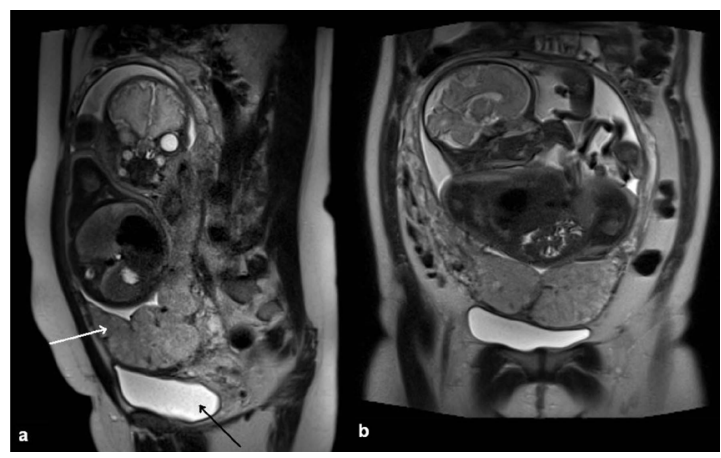

Figura 2. Resonancia nuclear magnética en placenta percreta. a) Corte sagital, ponderado en secuencia T2. Útero grávido con feto en su interior observando placenta percreta (flecha blanca), su inserción caudal en el útero y su relación con vejiga (flecha negra). b) Corte coronal, misma secuencia. 

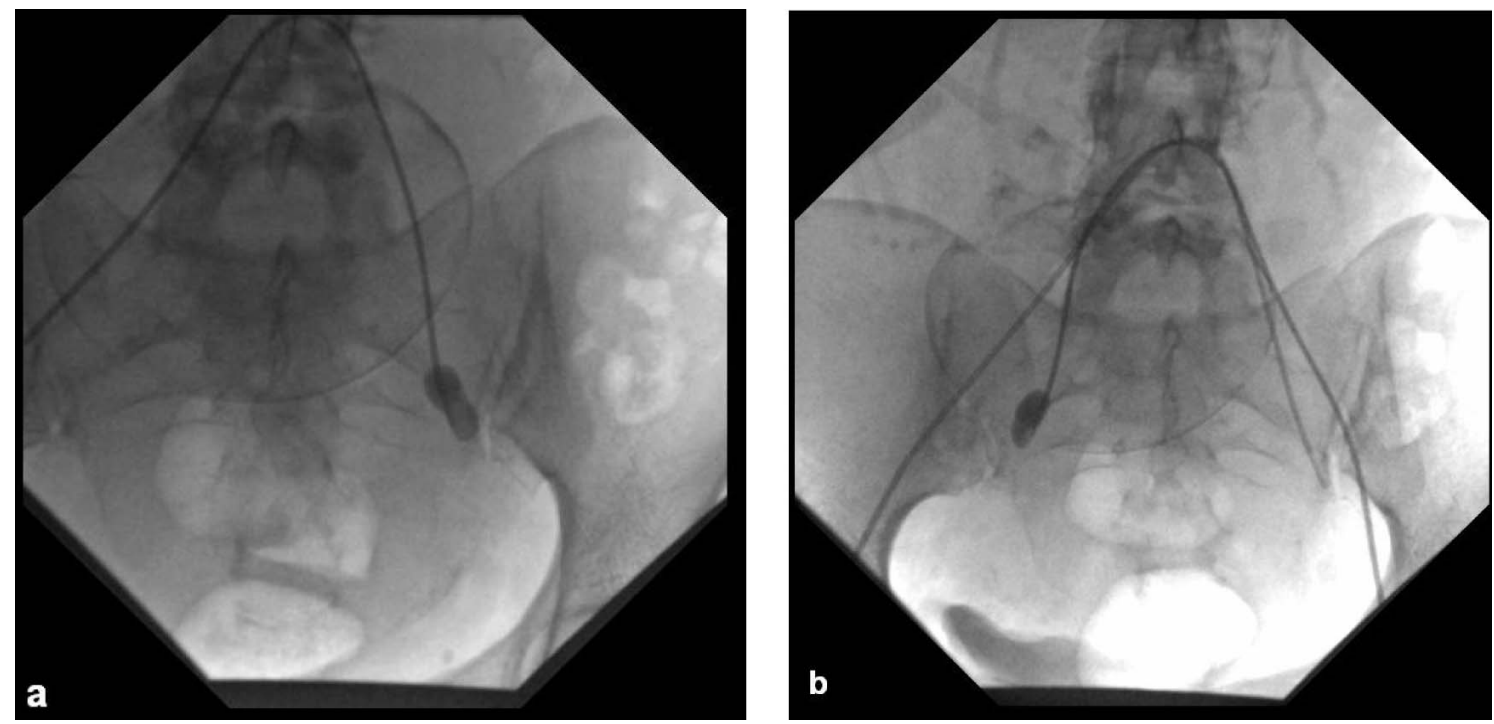

Figuras $3 a-b$. Visión angiográfica. Oclusión temporal de arterias hipogástricas. Insuflación de balón de oclusión, posicionado en ambas arterias hipogástricas.

El día de la intervención se efectuó: 1. Cateterización de arterias ilíacas internas con balones de oclusión en pabellón de angiografía (radiólogo intervencionista). Se punciona ambas arterias femorales con técnica de Seldinger, con catéteres Cobra 1 de 5 Fr., guías hidrofílicas y metálicas. Se avanzan los balones de oclusión selectivamente, posicionándolos en la división anterior de las arterias hipogástricas, contralateral al sitio de ingreso, fijándolos externamente con puntos a la piel y se traslada al pabellón quirúrgico. 2. Accesos vasculares, central y periférico, monitoreo invasivo y anestesia neuroaxial seguida de anestesia general posnacimiento (anestesiólogo) y 3. Citoscopia exploratoria con cateterización de uréteres (urólogo). Todos los procedimientos se efectuaron sin incidentes.

Cirugía: Laparotomía media infraumbilical, observándose adherencia vésico-uterina en cicatriz, con numerosos vasos prominentes, visibles subserosos y situados encima del límite vesical y miometrio ampliamente infiltrado en istmo por placenta (incretismo). Se continuó con histerotomía vertical alta, prefúndica, evitando la placenta, extracción del recién nacido vigoroso, ligadura de cordón a los 35 segundos, y oclusión bilateral simultánea de ilíacas por inflamiento de balones, histerorrafia en un plano, exteriorización uterina e histerectomía obstétrica con pinzamiento, doble sutura y sección de pedículos vasculares y elementos de sostén. La adherencia útero-vesical, vascularizada y laxa, permitió disección anterior con descenso de vejiga, apertura vaginal con extracción total del cuerpo y cuello uterino, sutura vaginal y puntos hemostáticos accesorios.

La operación duró dos horas y la paciente estuvo hemodinámicamente estable, con pérdida sanguínea estimada de 2000 cc. Se transfundieron $2000 \mathrm{cc}$ de coloides, 2500 de cristaloides y 1 unidad de glóbulos rojos. Los balones de oclusión insuflados en arterias ilíacas, se retiran previa evaluación imagenológica y quirúrgica buscando posibles puntos de sangrado durante el procedimiento; los catéteres ureterales fueron retirados a las 48 horas y la evolución posterior fue sin incidentes.

\section{DISCUSIÓN}

1. Detección antenatal de acretismo: Esto decide la estrategia de programar la cesárea-histerectomía sin remoción de placenta y con equipo multidisciplinario preparado. La carencia del diagnóstico al parto, sin proveer oportunamente los recursos apropiados, pone a la madre en grave riesgo de morbimortalidad.

Los signos ecográficos en escala de grises son: a) pérdida de la interfase uteroplacentaria, b) lagunas placentarias anormales, c) disrupción de la interfase vésico-uterina, d) prominencia de masa placentaria en la vejiga. Con el doppler color: a) flujo lacunar focal o difuso, b) hipervascularidad de interfase vesico-uterina, c) lagunas vasculares con flujo turbulento de alta velocidad y d) vasos dilatados, subplacentarios $(10,14,15)$. 
Estos signos reflejan neovascularización, profundidad y extensión de la invasión vellositaria e indirectamente el grado de adherencia y la magnitud esperada del sangrado. Pero por factores moleculares y respuesta individual (17) la invasividad varía caso a caso y aún en una misma paciente. Así, la adherencia anormal puede ser de profundidad variable (acretismo, incretismo o percretismo) y de extensión variable (acretismo focal o difuso) debiendo tener cada forma de invasión su expresión en las imágenes y en el sangrado, pero las biopsias no siempre representan el área de mayor invasión (3) y la expresión histopatológica de la adherencia no se ha validado en las imágenes.

El estudio histopatológico (patrón de oro) requiere secciones histológicas seriadas orientadas según aspecto macroscópico estimando con más exactitud la extensión y profundidad del compromiso miometrial y con ello reinterpretar mejor la imagen antenatal y su valor pronóstico, seleccionando casos de acretismo focal menor, tratables con manejo conservador.

Las lagunas vasculares placentarias son el signo más demostrativo con $93 \%$ de sensiblidad y $93 \%$ de valor predictivo positivo (14-16) y se asocia a complicaciones mayores. La sensibilidad y especificidad de las pruebas imagenológicas son altas en conjunto, pero disminuyen para cada signo individual con falsos positivos y falsos negativos. En nuestra experiencia inicial para el conjunto de signos se demostró sensibilidad de $81 \%$ y valor predictivo positivo de $76 \%$ (18).

En el caso descrito se sospechó acretismo por la observación de placenta previa central sobre dos cicatrices anteriores, y se evidenció por las lagunas placentarias grado 2 ( 3 a 6 por campo, grandes e irregulares cercanas a la placa materna) e hipervascularidad subplacentaria y de interfase utero-vesical. Estos signos nos hicieron pensar en invasión vesical, presente en 5 a $20 \%$ de las acretas, asociado a mortalidad materna de 10\% (19), con pérdida de sangre tres veces mayor que en placenta increta $(19,21)$ por lo que se planificó una interrupción preventiva utilizando técnicas de cateterización ureteral y oclusión de arterias ilíacas. El signo falso positivo de invasión vesical, correspondió a la imagen vascularizada del tabique proyectada a vejiga, pero fue descartada por la citoscopia y en la cirugía no hubo daño vesical.

La histopatología confirmó que las lagunas observadas correspondían a un incretismo que invadió ampliamente el miometrio en áreas de 10x7 cm en cara anterior y $12 \times 12 \mathrm{~cm}$ en cara posterior y percretismo que llegó a menos de $1 \mathrm{~mm}$ de la serosa uterina (Figura 4).

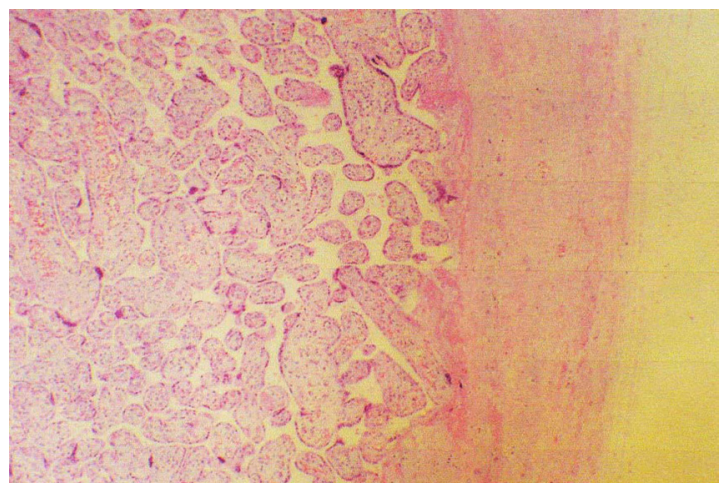

Figura 4. Microscopia de placenta con aumento $x$ 40.Tinción hematoxilina-eosina. Miometrio muy delgado a derecha, material fibrinoide y vellosidades coriales adheridas.

2. Oclusión de arterias ilíacas: Inicialmente exitosa en casuísticas pequeñas. La técnica está en discusión ya que Shrivastava y cols (20) en un estudio retrospectivo controlado en 69 pacientes, no demostró el beneficio de un menor sangrado en la cesárea-histerectomía con balón intra-arterial (19 casos). Pero en el grupo control sin balón (50 casos), hay un sesgo de selección con 9 pacientes (18\%) que no tenían cesárea previa, con menor riesgo de acretismo y menor posibilidad de sangrado, atenuando el beneficio esperado en el grupo de estudio. El autor describe complicaciones del procedimiento (3/19 o 15,8\%) (colocación, desplazamiento, remoción) dependientes del operador (experiencia previa, duración, número de intentos, trauma inadvertido).

En el caso presentado, el procedimiento fue bien tolerado, con cateterización selectiva de ambas ilíacas y sin embolización complementaria ni complicaciones posteriores. La insuflación de los balones efectuada inmediatamente al parto y mantenida durante la cirugía obtuvo una supresión de flujo suficiente ya que la paciente mantuvo estabilidad hemodinámica en ese período y la hemostasia quirúrgica fue satisfactoria, ya que al desinflar los balones no ocurrió un nuevo sangrado. La cuantía de la hemorragia intraoperatoria fue de $2000 \mathrm{cc}$, superior a los $1000 \mathrm{cc}$ propios de la cesárea habitual, pero muy menor a las pérdidas promedio descritas para la placenta percreta, de $12140 \pm 8343$ cc y para la placenta increta de $3630 \pm 2216$ cc (21). También se evitó la ligadura de las arterias ilíacas, más invasiva, con daño potencial de uréter y vena ilíaca interna, consumiendo además tiempo quirúrgico. 
Las técnicas utilizadas en cesárea-histerectomía por acretismo han demostrado resultados diferentes que se explican por la variabilidad en la invasión vellositaria y un espectro clínico amplio de gravedad.

Durante la oclusión, o posteriormente, puede persistir un sangrado, debido a extensas anastomosis vasculares colaterales descritas (22), que no ocurrió en el caso presentado. Otras causas de metrorragia incontrolable son la remoción placentaria, la dificultad quirúrgica en la hemostasia, la coagulopatía dilucional o por politranfusión y que pueden requerir otro procedimiento adicional.

La gravedad del cuadro hace improbable un estudio prospectivo con distribución al azar y controlado (5), las casuísticas son pequeñas y no hay fundamentos para recomendar una técnica única. En nuestro caso la oclusión permitió una cirugía sin apremio hemodinámico y esta experiencia sugiere adoptarla en el protocolo de manejo del acretismo extenso.

3. Enfoque multidisciplinario: La discusión multidisciplinaria se orienta a la posibilidad de hemorragia masiva, la elección del mejor procedimiento y el tratamiento postoperatorio. El acretismo invasivo puede comprometer órganos vecinos como vejiga, uréter e intestino y la cirugía reparadora debe ser apropiada minimizando su extensión y la morbilidad asociada, lo que justifica la participación de otros especialistas. La invasión vesical o del uréter, el daño ureteral espontáneo o iatrogénico, pueden requerir durante el parto operatorio cistectomía, cistorrafía, reparación vesical, reparación y/o neoimplante ureteral, haciendo indispensable la participación del urólogo, definida en el enfoque preoperatorio como cirujano del equipo o accesible de llamada. El radiólogo intervencionista, además de efectuar el procedimiento, define la duración y ampliación de la oclusión (embolización), evalúa resultado y complicaciones. Eventualmente puede ser necesaria la participación del cirujano vascular (9).

\section{CONCLUSIONES}

El enfoque multidisciplinario es una estrategia recomendable en cesárea-histerectomía por placenta acreta, que permite reducir el riesgo de morbimortalidad materna. Para lo anterior es fundamental el diagnóstico por imágenes del grado de invasión placentaria del útero y a los órganos vecinos

\section{REFERENCIAS}

1. Oyelese Y, Smulian JC. Placenta previa, placenta ac- creta and vasa previa. Obstet Gynecol 2006;107:92741.

2. Silver RM, Landon MB, Rouse DL, Leveno KJ, Spong $\mathrm{CY}$, Thon EA, et al. Maternal morbidity associates with multiple repeat cesarean deliveries. Obstet Gynecol 2006;107:1226-32.

3. Wu S, Kocherginsky M, Hibbard JU. Abnormal placentation: twenty - year analysis. Am J Obstet Gynecol 2005;1992:1458-61.

4. Eller AG, Porter TF, Soisson P, Silver RM: Optimal management strategies for placenta accreta. BJOG 2009;116:648-54.

5. Ramos GA, Kelly TF, Moore TR. The importance of preoperative evaluation in patients with risk factors for placenta accrete (abstract). Obstet Gynecol 2007;109: (4 Suppl): 7S.

6. Ng MK, Jack GS, Bolton DM, Lawrentschuk N. Female Urology. Placenta percreta with urinary tract involvement: the case for a multidisciplinary approach. Urology 2009;74:778-82 .

7. Palacios-Jaraquemada JM. Diagnosis and management of placenta accreta. Best Pract Res Clin Obstet Gynecol 2008;22:1133-48.

8. Hasbun J, Hidalgo G. Hemodynamic effects of placental delivery in placenta accreta. 3rd World Congress of Perinatal Medicine. San Francisco. EEUU. 1996. Book of Abstracs p 59.

9. Chou MM, Ke YM, Wu HC, Tsay CP, Ho ES, Ismail $\mathrm{H}$, Palacios Jaraquemada JM. Temporary cross-clamping of the infrarenal abdominal aorta during cesarean hysterectomy to control operative blood loss in placenta previa increta/percreta. Taiwan J Obstet Gynecol 2010;49:72-6.

10. ACOG Committee opinion. Number 266, January 2002: placenta accreta. ACOG Committee on Obstetric Practice. Obstet Gynecol 2002;99:169-70.

11. Weiniger CF, Elram T, Ginosar Y, Mankuta D, Weissman C, Ezra Y. Anaesthetic management of placenta accreta: use of a pre-operative high and low suspiction classification. Anaesthesia 2005; 60:1079-84.

12. Shih JC, Liu KL, Shyu MK. Temporary occlusion of the common iliac artery: new approach to bleeding control during a cesarean hysterectomy for placenta percreta. Am J Obstet Gynecol 2005;193:1756-8.

13. Angstmann T, Gard G, Harrington T, Ward E, Thompson Q, Giles W. Surgical management of placenta accreta: a cohort series and suggested approach. Am J Obstet Gynecol 2010:202:38 e1-9.

14. Comstock $\mathrm{CH}$. Antenatal diagnosis of placenta accreta: a review. Ultrasound Obstet Gynecol 2005;26:89-96.

15. Yang JL, Lim YK, Kim KH, Chang KH, Lee JP, Ryu HS. Sonographic findings of placental lacunae and the prediction of adherent placenta in women with placenta previa totalis and prior cesarean section. Ultrasound Obstet Gynecol 2006;28:178-82.

16. Woodring TC, Klauser CK, Bofill JA, Martin RW, Morrison JC. Prediction of placenta accreta by ultrasonography and color doppler imaging. J Matern Fetal Neonatal Med 2011;24:118-21.

17. Tseng JJ, Chou MM, Hsieh YT, Wen MC, Ho ES, Hsu SL. Differential expression of vascular endothelial 
growth factor, placenta growth factor and their receptors in placentae from pregnances complicated by placenta accreta. Placenta 2006; 27:70-8.

18. Valdés E,Chahuan B, Hasbun J, Parra M, Atria A. Utilidad del examen ultrasonográfico en el diagnóstico del acretismo placentario. XXXI Congreso Chileno de Obstetricia y Ginecología 2007. Libro de Resúmenes. pp 82.

19. Hudon L, Belfort MA, Broome DR. Diagnosis and management of placenta percreta: a review. Obstet Gynecol Surv 1998;53:509-17.

20. Shrivastrava V, Nageotte M, Major C, Haydon M, Wing D. Case-control comparison of cesarean hys- terectomy with and without prophylactic placement of intravascular balloon catheters for placenta accreta. Am J Obstet Gynecol 2007;197:402.e1-5.

21. Sumigama $S$, Ikakura $A$, Ota $T$, Okada M, Kotany $T$, Hayakawa $\mathrm{H}$, et al. Placenta increta/percreta in Japan: a retrospective study of ultrasound findings, management and clinical course. J Obstet Gynecol Res 2007;33:606-11.

22. Palacios Jaraquemada JM, García Mónaco R, Barbosa NE, Ferle L, Iriarte H, Conesa HA. Lower uterine blood supply: extrauterine anastomotic system and its application in surgical devascularization techniques. Acta Obstet Gynecol Scand 2007;86:228-34. 